\title{
A Classification of Snow Clouds by Doppler Radar Observations at Nagaoka, Japan
}

\author{
Sento Nakai ${ }^{1}$, Koyuru Iwanami ${ }^{2}$, Ryohei Misumi ${ }^{2}$, Sang-Goon Park ${ }^{2}$, and Toshiichi Kobayashi ${ }^{1}$ \\ ${ }^{1}$ Snow and Ice Research Group, NIED, Nagaoka, Japan \\ ${ }^{2}$ Advanced Technology Research Group, NIED, Tsukuba, Japan
}

\begin{abstract}
A classification of snow clouds, called the "snowfall mode," is proposed based on Doppler radar observations at 10-minute intervals at Nagaoka in 1999/2000 winter season. Using 795 hours of data at an altitude of $1.6 \mathrm{~km}$, six snowfall modes were defined: longitudinal line (Lmode), transversal line (T-mode), spreading precipitation (S-mode), meso- $\beta$ scale vortex (V-mode), mountainslope precipitation (M-mode), and local-frontal (discontinuity) band (D-mode). In migrating snow clouds, a subclass, referred to as snowfall with coastal intensification (xI-mode, where $\mathrm{x}$ is $\mathrm{L}, \mathrm{T}, \mathrm{S}$ and $\mathrm{V}$ ) was defined. A sample snapshot and the mean $Z e$ are shown for each snowfall mode. The frequency of occurrence of the snowfall modes indicated that both of the longitudinal cloud streets and the meso- $\alpha$ scale disturbances occupied about $1 / 3$ of the analysis period. About $18 \%$ of the precipitation in the analysis period was considered to be under orographic effects. The prevailing wind direction differed between the snowfall modes although a west-northwesterly wind dominated.
\end{abstract}

\section{Introduction}

Snow clouds developing over the Sea of Japan generate a wide variety of radar echo patterns, which suggests that there are a number of mechanisms involved in the development of snow clouds. In the 1970s, some classifications of snow clouds were made using conventional radars (e.g., Nanasawa 1975). However, their time resolution was low that the motion and duration of the specific patterns were not analyzed. Since then, many theoretical and observational case studies have been conducted. There are several wellknown structures of snow clouds. Longitudinal (Lmode) snowbands often correspond to "cloud streets" that appear during cold outbreaks. The structure of the transversal (T-mode) snowbands was recently elucidated (Murakami et al. 2002). Vortex disturbances often appear around the Japan Sea Polar-Airmass Convergence Zone (JPCZ) (Asai 1988; Tsuboki and Asai 2004). They were also observed as radar echoes (e.g., Asai and Miura 1981). Moreover, land breezes contribute to the formation of snowbands and significantly affect the snowfall (e.g., Ishihara et al. 1989; Ohigashi and Tsuboki 2005).

Thus, various structure and development processes of the snow clouds have been analyzed. However, systematic morphological terminology has not been established, and the frequency of occurrence has not been thoroughly analyzed.

The Nagaoka Institute of Snow and Ice Studies (NISIS) locates in the central part of the Niigata Prefecture (Fig. 1). The NISIS makes it possible to

Corresponding author: Sento Nakai, Nagaoka Institute of Snow and Ice Studies, National Research Institute for Earth Science and Disaster Prevention, Suyoshi, Nagaoka 940-0821, Japan. E-mail: saint@bosai.go.jp. (02005, the Meteorological Society of Japan.

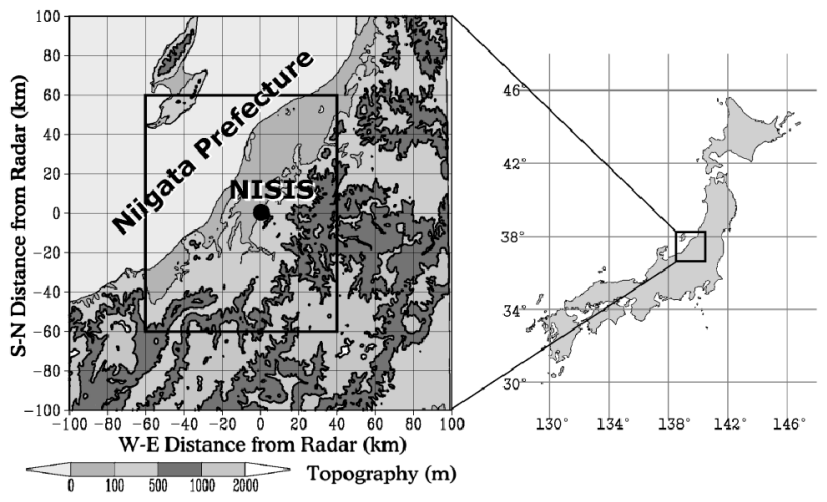

Fig. 1. Location of the NISIS. The rectangle enclosed by thick lines in the left panel is the area shown in Figs. 3 and 4.

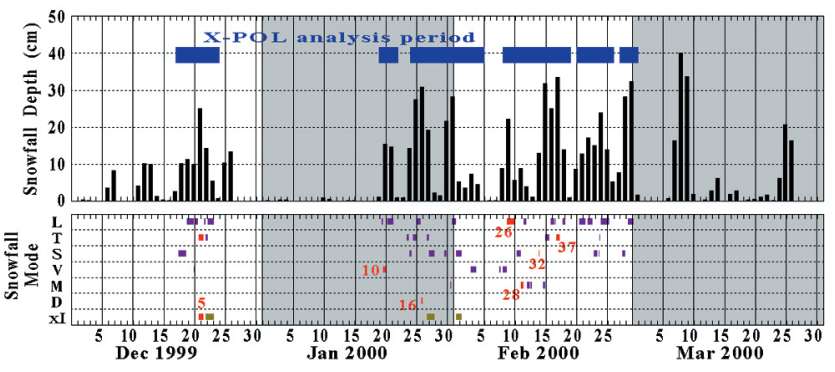

Fig. 2. Daily snowfall depth (from 09 JST on a day to 09 JST on the next day) averaged for 22 observation sites of Niigata Prefecture within $60 \mathrm{~km}$ from X-POL. The analysis period of the X-POL data is shown in thick blue bars. The duration of the 46 cases were indicated by the bars in the lower panel. The cases shown in Figs. 3 and 4 are indicated in red with the case number.

observe snow clouds throughout the winter season. In this paper, we propose a classification of snow clouds or "snowfall modes" based on Doppler radar winter observations.

\section{Observation}

An X-band Doppler radar, X-POL (Iwanami et al. 1996), was set up on the roof of the NISIS. The observation area was a northwestern-side semicircle with a radius of $64 \mathrm{~km}$. The radar operation consisted of 15 steps of a PPI scan, repeated at about 10-minute intervals. Three-dimensional distributions of the equivalent radar reflectivity factor $(Z e)$ and radial velocity $(V r)$ were obtained. The radar sampling resolution was 250 $\mathrm{m}$ in range and 0.703 degree in azimuth. The observed $Z e$ and $V r$ on a polar coordinate were converted to a Cartesian coordinate with horizontal and vertical resolutions of $1 \mathrm{~km}$ and $500 \mathrm{~m}$, respectively.

We made 44 days of observations from December 1999 through March 2000 (thick blue lines in Fig. 2), 
Table 1. Classification of snow clouds

\begin{tabular}{|l|l|}
\hline Longitudinal line (L-mode) & Bands running or cells aligned nearly parallel to the prevailing wind \\
\hline Transversal line (T-mode) & Bands running or cells aligned with a large angle relative to the prevailing wind \\
\hline Spreading precipitation (S-mode) & Widely spreading, relatively uniform precipitation \\
\hline Meso- $\beta$ scale vortex (V-mode) & Vortices and associated curved bands with a significant change of the wind direction \\
\hline Mountain-slope precipitation (M-mode) & Area of stationary precipitation around the windward slope of the mountains \\
\hline Local-frontal band (D-mode) & A wide band that considered to be formed along a line of discontinuity \\
\hline sub-class of the modes shown above & \multicolumn{2}{|l}{} \\
\hline Coastal intensification (xI-mode) & Precipitation occasionally intensified around the coastline \\
\hline
\end{tabular}

and obtained 795 hours of radar precipitation data. The data included most of the snowfall in January and February 2000, and a major snowfall event in December 1999 (Fig. 2).

\section{Classification of snow clouds}

Animation of the horizontal section of $\mathrm{Ze}$ and $\mathrm{Vr}$ at an altitude of $1.6 \mathrm{~km}$ were used for the classification. If a characteristic radar echo pattern continued in the animation, it was considered as a case, then, the cases were classified into snowfall modes. The classification was conducted mainly using $Z e$ with attention given to 1 ) radar-echo characteristics in the entire area under analysis, and 2) specific patterns that continued for a specific period of time. The prevailing wind direction of each case was decided mainly by $V r$ patterns on the horizontal sections at an altitude of $1.6 \mathrm{~km}$, which was a typical steering level of the snow clouds. The $V r$ was also used supplementary for the classification.

Forty-six cases were defined among 795 hours of animation. The distributions of the mean $Z e$ were also derived for each case. We found seven characteristic $Z e$ patterns, which are called as "snowfall modes" (Table 1). Among them, six are major classes, and the other one is a sub-class describing additional characteristics. The lower panel of Fig. 2 shows the result of the classifica- (a) Longitudinal line (L)

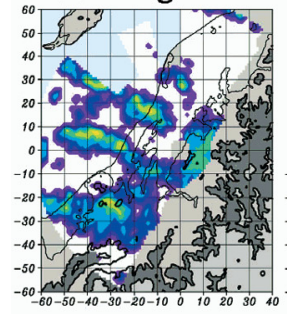

(d) Meso- $\beta$ scale vortex (V)
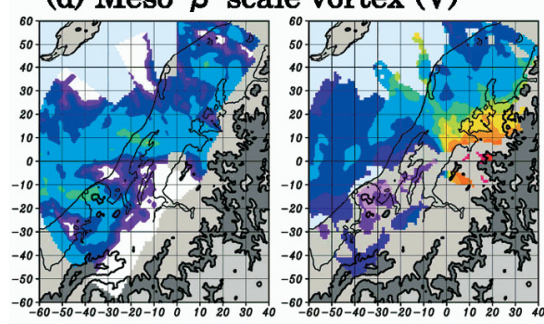

(g) Trasversal line with coastal intensification (TI)
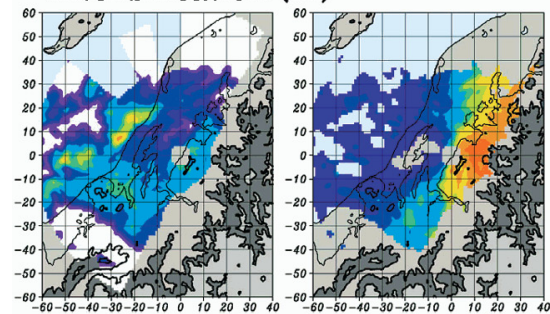

(b) Transversal line (T)

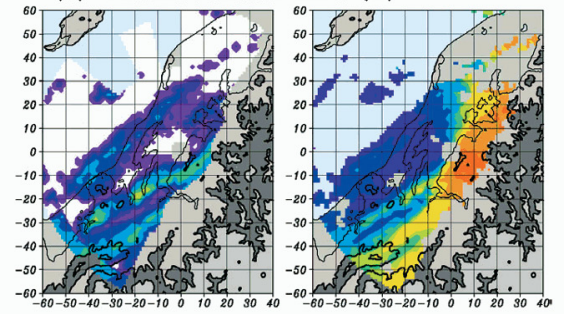

(e) Mountain-slope precipitation (M)
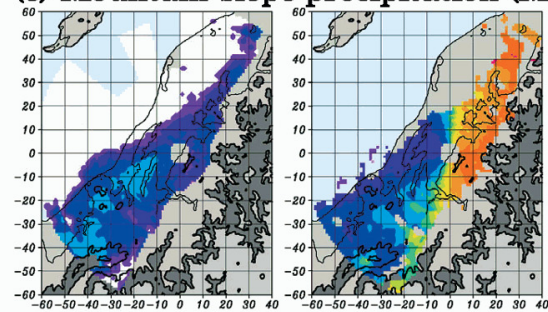

(c) Spreading precipitation (S)
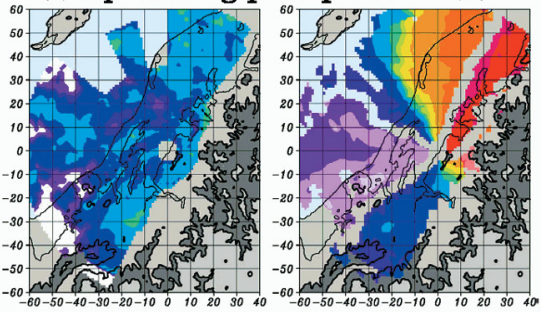

(f) Local-frontal band (D)
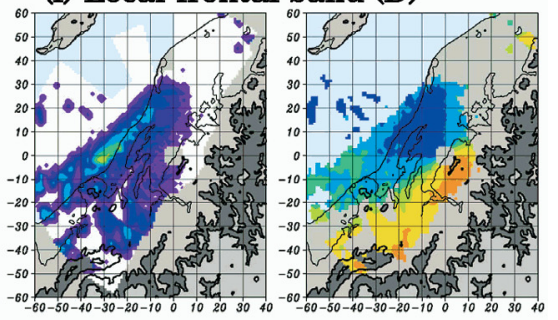

Legend

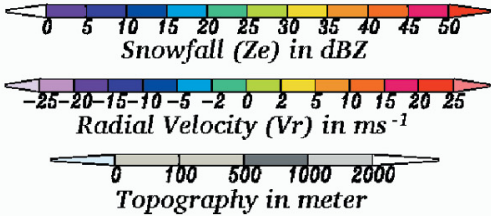

Fig. 3. Examples of the snowfall modes shown in Table 1. Seven pairs of horizontal sections of $Z e$ (left) and $V r$ (right) at $1.6 \mathrm{~km}$ above sea level are shown: (a) longitudinal line (L-mode, case 26) at 1210 JST on 10 February 2000, (b) transversal line (T-mode, case 37) at 2122 JST on 17 February 2000, (c) spreading precipitation (S-mode, case 32) at 2032 JST on 14 February 2000 , (d) meso- $\beta$ scale vortex (V-mode, case 10) at 1940 JST on 20 January 2000 , (e) mountain-slope precipitation (M-mode, case 28 ) at 0147 JST on 12 February 2000, (f) local-frontal band (D-mode, case 16) at 2110 JST on 26 January 2000, and (g) transversal line with coastal intensification (TI-mode, case 5) at 0840 JST on 22 December 1999. The ordinate and the abscissa are the east-west and south-north distances from X-POL, respectively, in km. 

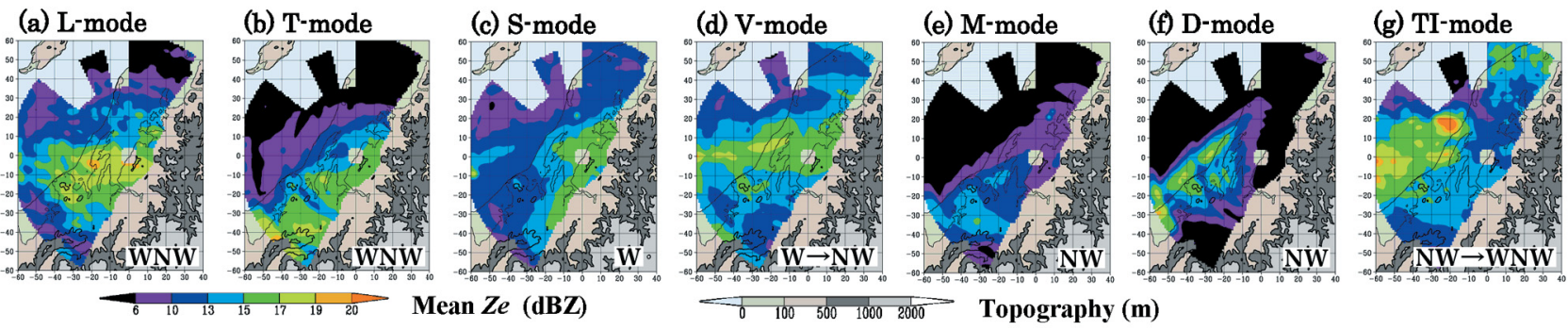

Fig. 4. Examples of the mean $Z e$ at $1.6 \mathrm{~km}$ above sea level of (a) longitudinal line (L-mode, case $26,29.1 \mathrm{hours),} \mathrm{(b)} \mathrm{transversal}$ line (T-mode, case 37, 12.7 hours), (c) spreading precipitation (S-mode, case 32, 3.9 hours), (d) meso- $\beta$ scale vortex (V-mode, case 10, 10.5 hours), (e) mountain-slope precipitation (M-mode, case 28, 9.0 hours), (f) local-frontal band (D-mode, case 16, 4.0 hours), and $(\mathrm{g})$ transversal line with coastal intensification (TI-mode, case 5, 19.8 hours). The ordinate and the abscissa are the east-west and south-north distances from X-POL, respectively, in $\mathrm{km}$. The circular patterns centered at the origin are artificial ones caused by the gap of elevation of the original radar data on the polar-coordinate. The prevailing wind direction is shown at the lower right corner of each panel.

tion of the 46 cases. Examples of the snowfall modes are shown in Fig. 3. For the cases including the snapshot shown in Fig. 3, the mean $Z e$ of the case are shown in Fig. 4. The mean $Z e$ was calculated at each grid point of the Cartesian coordinate by $(1 / \mathrm{N}) \Sigma Z e$, where $\mathrm{N}$ is the number of the Cartesian grid data every 10 minutes and $Z e$ is in real number. The summation represents the duration period of the case.

Two of the snowfall modes are the well-known "Lmode" (Fig. 3a) and "T-mode" (Fig. 3b), that is, lines longitudinal and transversal to the prevailing wind, respectively. A line is a snowband or is sometimes composed of separate cells. The prevailing wind direction is perpendicular to the contour of $V r=0$ and west-northwest in the right panels of Figs. $3 \mathrm{a}$ and $3 \mathrm{~b}$. The running direction of a snowband or a chain of cells is nearly parallel (perpendicular) to the prevailing wind direction in Fig. 3a (3b).

The mean $Z e$ of the L-mode snow cloud was characterized by local maxima extending from the sea to the inland area (Fig. 4a). The maxima were formed by the consecutive passage of snowbands through a certain path. The direction of motion of the L-mode snow clouds was close to the prevailing wind direction, so the precipitation distribution was strongly affected by the path of the snow clouds. The mean $Z e$ distribution of Tmode snow clouds had inland maxima (Fig. 4b), although radar echoes were seen over land and sea (Fig. $3 \mathrm{~b})$. The result indicates that the T-mode snow clouds moved inland, adding precipitation intensity.

The spreading precipitation (S-mode) is characterized by continuous, rather uniform precipitation. The $Z e$ distribution of Fig. 3c indicates that precipitation covered most of the X-POL observation range. The echo gap $(<0 \mathrm{dBZ})$, as in Figs. $3 \mathrm{a}$ and $3 \mathrm{~b}$, is not seen in Fig. 3c. Moderate values also spread widely in the mean $Z e$ distribution of the S-mode (Fig. 4c) as compared to the Land $\mathrm{T}$ - modes.

The meso- $\beta$ scale vortex ( $\mathrm{V}$-mode) indicates one or a sequence of vortices with a diameter of $20 \mathrm{~km}-100 \mathrm{~km}$ including curved snowbands that appear before or after the vortices. The example (Fig. 3d) shows a vortex located at $30 \mathrm{~km}$ west from X-POL with a diameter of about $50 \mathrm{~km}$. All vortices recognized in the animation rotated counterclockwise. A significant change in the prevailing wind direction was often observed accompanying the passage of the vortices. The mean $Z e$ of the $\mathrm{V}$ mode had maxima along the path of the vortices (Fig. 4d).

The mountain-slope precipitation (M-mode) means that stationary weak precipitation was continuously observed around the windward slope of the mountains. In Fig. 3e, the $V r$ distribution shows that the wind direction was west-northwest; however, the precipitation shown by $Z e$ was almost stationary and did not migrate from the sea. Therefore, the mean $Z e$ (Fig. 4e) showed a distribution similar to the snapshot $Z e$ distribution (Fig. $3 e)$. It is noteworthy that no precipitation was observed upwind over the sea. The M-mode precipitation was considered to have been formed by a strong orographic effect on the originally non-precipitating snow clouds.

The local-frontal (discontinuity) band (D-mode) indicates a snowband stationary or oscillating off or around the coastline. The snowband shown in Fig. 3f was stationary, and the mean $Z e$ distribution (Fig. 4f) was very similar to the snapshot $Z e$ distribution. The magnitude of the snapshot $Z e$ and the mean $Z e$ was much larger than that of the M-mode. Such a snowband is usually formed along a local front (discontinuity) between the monsoon wind and the land breeze. The land breeze was observed at the surface in the D-mode case shown in Figs. 3f and $4 \mathrm{f}$.

Some cases of migrating snow clouds (L-, T-, S- and $\mathrm{V}$-modes) included precipitation that intensified around the coastline. They are grouped into an xI-mode, where $\mathrm{x}$ is one of the above-mentioned snowfall modes. For example, the T-mode with coastal intensification is called the TI-mode. LI-, TI-, and SI-mode cases were found during the analysis period. In Fig. 3g, a T-mode snowband, located at $10 \mathrm{~km}$ off the coastline, was significantly intensified. The snowband weakened as it migrated inland, suggesting that the snowband was intensified by an orographic effect. The mean $Z e$ reflected this variation of precipitation intensity (Fig. $4 \mathrm{~g}$ ).

\section{Frequency of occurrence}

The number of cases, the cumulative observed duration time (CDT) and its percentage to the total observed precipitation time (\%CDT) were calculated for each snowfall mode (Table 2 ).

The L-mode dominated in the number of cases, and occupied $1 / 3$ of the analysis period as indicated by \%CDT. The L-mode snow clouds are usually longitudinal cloud streets. They appear widely over the Sea of Japan so that the high frequency of occurrence of the L-mode is reasonable. The \%CDT of the S- and T-modes were $17 \%$ and $12 \%$, respectively, and the CDT of the V-and $\mathrm{M}$ modes were short. Only one case was observed, as for the D-mode. The sum of the \%CDT was $79 \%$, indicating that we did not recognize a specific Ze pattern in $20 \%$ of the precipitation in the analysis period. Moreover, some cases suffered from the lack of data, resulting in the artificially shortened duration time. Therefore, we confine the discussion on the frequency to some qualitative points. 
Table 2. Number of cases (\# cases), cumulative observed duration time (CDT in hour) and its percentage to the total observed precipitation time (\%CDT) for the snowfall modes appeared during the analysis period. Values in the parentheses are for the xI-modes.

\begin{tabular}{|c|c|c|c|c|c|c|c|}
\hline Mode & $\mathrm{L}(\mathrm{LI})$ & $\mathrm{T}(\mathrm{TI})$ & $\mathrm{S}(\mathrm{SI})$ & $\mathrm{V}$ & $\mathrm{M}$ & $\mathrm{D}$ & Total \\
\hline \# cases & $17(1)$ & $8(3)$ & $10(2)$ & 5 & 5 & 1 & 46 \\
\hline CDT & $297(24)$ & $93(35)$ & $137(39)$ & 58 & 37 & 4 & 625 \\
\hline \%CDT & $37(3)$ & $12(4)$ & $17(5)$ & 7 & 5 & 1 & 79 \\
\hline
\end{tabular}

$\mathrm{V}$ - and T-mode snow clouds are associated with the JPCZ, and the S-mode snow clouds are associated with the JPCZ and fronts (Nakai et al. 1998; Nakai et al. 2004). In other words, the V-, T-, and S-mode snow clouds are related to meso- $\alpha$ scale disturbances. The sum of the $\%$ CDT of the three snowfall modes was $36 \%$, almost the same as that of the longitudinal cloud streets. The M-, Dand xI-modes are considered to be influenced by the topography (including coastal effect), and the sum of the $\%$ CDT of these modes was $18 \%$. It is half of the \%CDT of the L-mode snow clouds and also that of the snow clouds associated with meso- $\alpha$ scale disturbances.

According to the $V r$ at an altitude $1.6 \mathrm{~km}$, a westnorthwest wind prevailed (not shown). In particular, the prevailing wind direction during the L- and T-mode cases concentrated on the west-northwest directions. The L-mode snowbands (or lines of cells) usually correspond to the cloud streets longitudinal to the monsoon wind, where the typical direction is northwest. The Lmode prevailing wind direction suggests that the deflection of the wind may often occur around the coastline of the Niigata Prefecture. Most of the coastal snowfall intensification also occurred under west-northwesterly prevailing wind. M- and D- mode snowfall was mainly observed with a northwesterly prevailing wind. The Smode snow clouds tended to appear with a westerly prevailing wind. The V-mode snow clouds were accompanied by a significant clockwise change in the prevailing wind direction starting from west-southwest directions. The veering occurred when a major vortex passed the X-POL observation area.

\section{Summary}

We conducted Doppler radar observations through the 1999/2000 winter season at Nagaoka. A classification of snow clouds was made based on the time sequence of the $Z e$ and $V r$ patterns that appeared in the animation of a horizontal section at a height of $1.6 \mathrm{~km}$. The snow clouds were classified into six snowfall modes and one sub-class as shown in Table 1. The characteristics of the snowfall modes were described with examples of the $\mathrm{Ze}$ and $\mathrm{Vr}$ distributions (Fig. 3). Each snowfall mode had a characteristic mean $Z e$ pattern (Fig. 4).

All of the longitudinal cloud streets (the L- mode), meso- $\alpha$ scale disturbances (the T-, S-, and V-modes) and orographically influenced snowfall (the M-, D-, and xImodes) contributed to the duration time of snowfall in the observation area significantly. The frequency of prevailing wind direction showed a maximum at westnorthwest and distributed mainly from west through northwest except in the cases of the S- and V-modes. They often appeared with a prevailing wind direction ranging from west through southwest.

The radar data used for the classification in this paper had Doppler wind information and much higher time resolution than that in the previous studies. This feature enable us to find xI-mode, and distinguish Mand D- modes. On the other snowfall modes, found in the previous studies, we related them to the duration time of snowfall and to the prevailing wind aloft. Previous studies discussed the relation between the snowfall modes and the synoptic situation, such as the location of the synoptic low pressure center. However, smaller-scale disturbances and orographic effects should be additional conditions to decide the meso- $\beta$ scale structure of snow clouds. To investigate the complex snowfall processes over and near land, Doppler radar data with high time resolution is essential. Characteristic airflow structure and precipitation mechanism related to the snowfall modes are remaining topics. The statistical and time-series analyses of vertical profiles of wind and $\mathrm{Ze}$ based parameters, including vertical wind shear, are necessary, as well as the case analyses and modeling studies.

\section{Acknowledgments}

The authors thank Mr. Masujiro Shimizu for his cooperation in the observations. Two anonymous reviewers gave us valuable comments. The Niigata Prefecture provided the daily snowfall data. This work is supported by two projects of the National Research Institute for Earth Science and Disaster Prevention titled "Research on possibility prediction system of snow disasters" and "Study on future changes in the global hydrologic cycle and related disasters."

\section{References}

Asai, T., and Y. Miura, 1981: An analytical study of meso-scale vortex-like disturbances observed around Wakasa Bay area. J. Meteor. Soc. Japan, 59, 832-843.

Asai, T., 1988: Mesoscale features of heavy snowfalls in Japan Sea coastal regions of Japan (in Japanese). Tenki, 35, 156161 .

Ishihara, M., H. Sakakibara and Z. Yanagisawa, 1989: Doppler radar analysis of the structure of mesoscale snow bands developed between the winter monsoon and the land breeze. J. Meteor. Soc. Japan, 67, 503-520.

Iwanami, K., M. Maki, T. Sato and M. Higashiura, 1996: Distribution of precipitation parameters estimated from observations with a Doppler radar and a polarimetric radar: Part 2, Results from polarimetric radar observation. Proceedings, 12th international conference on clouds and precipitation, 190-192.

Murakami, M., M. Hoshimoto, N. Orikasa, H. Horie, H. Okamoto, H. Kuroiwa, H. Minda and K. Nakamura, 2002: Inner structures of snow bands associated with the Japan Sea polarairmass convergence zone based on aircraft observations. Proceedings, international conference on mesoscale convective systems and heavy rainfall/snowfall in East Asia, 522527.

Nakai, S., M. Kajikawa and Y. Yamada, 1998: The relation between hydrometeor type and radar echo structure around the Dewa Hills. Atmos. Res., 47-48, 97-112.

Nakai, S., M. Ishizaka, K. Iwamoto, M. Shimizu, A. Sato and T. Maruyama, 2004: An observational study of the Niigata snowstorm of 4-6 January 2003. Proceedings, 14th international conference on clouds and precipitation, 1715-1718.

Nanasawa, K., 1975: Radar echo patterns classified by synoptic situations for the snowfall forecast for Central Hokkaido (in Japanese). J. Meteor. Res., 27, 177-188.

Ohigashi, T., and K. Tsuboki, 2005: Structure and maintenance process of stationary double snowbands along the coastal region. J. Meteor. Soc. Japan, 83, 331-349.

Tsuboki, K., and T. Asai, 2004: The multi-scale structure and development mechanism of mesoscale cyclones over the Sea of Japan in winter. J. Meteor. Soc. Japan, 82, 597-621.

Manuscript received 15 June 2005, accepted 10 August 2005 SOLA: http://www.jstage.jst.go.jp/browse/sola/ 